\title{
Fourth-order stable central difference method for self-adjoint singular perturbation problems
}

Terefe Asrat, Gemechis File* and Tesfaye Aga

Department of Mathematics, Jimma University, Jimma, Ethiopia

\begin{abstract}
In this paper, fourth order stable central difference method is presented for solving self-adjoint singular perturbation problems for small values of perturbation parameter, $\varepsilon$. First, the given differential equation was reduced to its conventional form and then it was transformed into linear system of algebraic equations in the form of a three-term recurrence relation, which can easily be solved by using Thomas Algorithm. To validate the applicability of the method, four model examples have been solved for different values of perturbation parameter and mesh sizes. The numerical results are tabulated and compared with some of the previous findings reported in the literature and it is observed that the present method is more efficient. Graphs are also depicted in support of the numerical results. Both theoretical error bounds and numerical rate of convergence have been established for the method.
\end{abstract}

Key Words/Phrases: Singular perturbation; stable central difference method; self-adjoint.

DOI: http://dx.doi.org/10.4314/ejst.v9i1.5

\section{INTRODUCTION}

Any differential equation obtained from a given differential equation and having the property that its solution is an integrating factor of the other is known as adjoint differential equation. A differential equation that has the same solution as its adjoint differential equation is known as self-adjoint differential equation and if it's highest order derivative is multiplied by a small positive parameter, $\varepsilon(0<\varepsilon<1)$, which has the form: $-\varepsilon\left(p(x) y^{\prime}\right)^{\prime}+q(x) y=f(x)$ is called second order self-adjoint singular perturbation problem (Byme and Mishra, 2009).

In finite difference methods, derivatives appearing in the differential equations are replaced by finite difference approximations obtained by Taylor series expansions at the grid points. This gives a large algebraic system of equations to be solved by any iterative methods in place of the differential equation to give the solution at the grid points and hence the solution is obtained at grid points. It is well-known that standard discretization methods for solving self-adjoint singular perturbation problems are unstable and fail to give accurate results when the perturbation parameter $\varepsilon$ is very small.

Classical, computational approaches to singularly perturbed problems are known to be inadequate as they require extremely large numbers of mesh points to produce satisfactory solutions (Roos et al., 1996; Farell et al., 2000). Detailed discussions on the theory of asymptotical and numerical solutions of singular perturbation problems have been published (Boglave, 1981; Kadalbajoo and Kumar, 2008; Mishra et al, 2009; Gupta and Pankaj, 2011). So, the treatment of singularly perturbed problems presents severe difficulties that have to be addressed to ensure accurate numerical solutions (Roos et al., 1996; Kadalbajoo and Kumar, 2010).

\footnotetext{
*Corresponding author: gammeef@yahoo.com

(C) This is an Open Access article distributed under the terms of the Creative Commons Attribution License (http://creativecommons.org/licenses/CC BY4.0).
} 
Further, these authors proved that the stability of three-points in higher order stable central difference method is $\varepsilon$-independent. Their aim was to devise numerical schemes with constant mesh spacing $h \geq \varepsilon$ to yield accurate solutions. However, they were able to show in general, that the accuracy of the scheme cannot be better than $o\left(h^{2}\right)$. Moreover, (Saini and Mishra ,2015) presented an algorithm to develop approximate solution of third-order self-adjoint singularly perturbed two-point boundary value problem in which the highest order derivative is multiplied by a small parameter. First, they introduce the Quartic B-spline basis function, and then they use the linear sequence of Quartic B-spline to get the numerical solution of system of equations. Thus, existing numerical methods produce good results only when we take step size $h<\varepsilon$, but this gives us a very large number of systems of equations that may require high capacity machines/computers or more time to run and to get the results easily.

In this paper, we present fourth order stable central difference method that is accurate and easy for solving self-adjoint singularly perturbed two-point boundary value problems for small values of perturbation parameter $\varepsilon$. Both theoretical error bounds and computational rate of convergence have been established.

\section{DESCRIPTION OF THE METHOD}

Consider the following self-adjoint singularly perturbed equationb of the form:

$-\varepsilon\left(p(x) y^{\prime}\right)^{\prime}+q(x) y(x)=f(x) \quad 0<x<1$

with the Dirichlet boundary conditions,

$y(0)=\alpha, y(1)=\beta$

where, $\varepsilon(0<\varepsilon<1)$ is a small parameter; $\alpha, \beta$ are given constants and $p(x), q(x)$ and $f(x)$ are assumed to be sufficiently continuous differentiable functions. To describe the method, we divide the interval $[0,1]$ into $N$ equal subintervals of mesh length $h$. Let $0=x_{0}, x_{1}, x_{2}, \ldots, x_{N}=1$ be the mesh points. Then, we have $\mathrm{x}_{\mathrm{i}}=\mathrm{x}_{0}+\mathrm{ih}, i=0,1,2, \ldots ., N$

Rewriting Eq. (1) at $x=x_{i}$, we have:

$-\varepsilon\left(p\left(x_{i}\right) y^{\prime}\left(x_{i}\right)\right)^{\prime}+q\left(x_{i}\right) y\left(x_{i}\right)=f\left(x_{i}\right) 0 \leq x_{i} \leq 1, i=0,1,2, \ldots ., N$

with boundary conditions : $y\left(x_{o}\right)=\alpha$ and $y\left(x_{N}\right)=\beta$

It can also be rewritten in the form:

$-y_{i}^{\prime \prime}+a\left(x_{i}\right) y^{\prime}\left(x_{i}\right)+b\left(x_{i}\right) y\left(x_{i}\right)=c\left(x_{i}\right)$

where, $a\left(x_{i}\right)=\frac{-p^{\prime}\left(x_{i}\right)}{p\left(x_{i}\right)}, \quad b\left(x_{i}\right)=\frac{q\left(x_{i}\right)}{\varepsilon p\left(x_{i}\right)}$ and $c\left(x_{i}\right)=\frac{f\left(x_{i}\right)}{\varepsilon p\left(x_{i}\right)}$

For the sake of simplicity, let us denote $a\left(x_{i}\right)=a_{i}, \quad b\left(x_{i}\right)=b_{i} \quad c\left(x_{i}\right)=c_{i}$, $y^{\prime}\left(x_{i}\right)=y_{i}^{\prime}, \quad y^{\prime \prime}\left(x_{i}\right)=y_{i}^{\prime \prime}$ and $y^{(n)}\left(x_{i}\right)=y_{i}^{(n)}$. 
To find a description of fourth order stable central difference scheme, we use Taylor's series expansion in order to get central difference formula for $y_{i}^{\prime \prime}$ and $y_{i}^{\prime}$. Assume that $y_{i}$ has continuous fourth derivatives in the interval $[0,1]$.

$y_{i+1}=y_{i}+h y_{i}{ }^{\prime}+\frac{h^{2}}{2 !} y_{i}{ }^{\prime \prime}+\frac{h^{3}}{3 !} y_{i}{ }^{\prime \prime \prime}+\frac{h^{4}}{4 !} y_{i}^{(4)}+\frac{h^{5}}{5 !} y_{i}^{(5)}+\frac{h^{6}}{6 !} y_{i}^{(6)}+O\left(h^{7}\right)$

$y_{i-1}=y_{i}-h y_{i}{ }^{\prime}+\frac{h^{2}}{2 !} y_{i}^{\prime \prime}-\frac{h^{3}}{3 !} y_{i}^{\prime \prime \prime}+\frac{h^{4}}{4 !} y_{i}^{(4)}-\frac{h^{5}}{5 !} y_{i}^{(5)}+\frac{h^{6}}{6 !} y_{i}^{(6)}+O\left(h^{7}\right)$

From Eqs. (4) and (5), we obtain the central difference approximation for the first and second derivative of $y_{i}$ as:

$y_{i}^{\prime}=\frac{y_{i+1}-y_{i-1}}{2 h}-\frac{h^{2}}{6} y_{i}{ }^{\prime \prime}+\tau_{1}$

where, $\quad \tau_{1}=-\frac{h^{4} y_{i}^{(5)}}{120}+0\left(h^{6}\right)$

$$
y_{i}^{\prime \prime}=\frac{y_{i+1}-2 y_{i}+y_{i-1}}{h^{2}}-\frac{h^{2}}{12} y_{i}^{(4)}+\tau_{2}
$$

where, $\quad \tau_{2}=-\frac{h^{4}}{360} y_{i}^{(6)}+0\left(h^{6}\right)$

Substituting Eqs. (6) and (7) into Eq. (3) at $x=x_{i}$, we obtain:

$$
\begin{gathered}
\left(\frac{1}{h^{2}}-\frac{a_{i}}{2 h}\right) y_{i-1}+\left(b_{i}-\frac{2}{h^{2}}\right) y_{i}+\left(\frac{1}{h^{2}}+\frac{a_{i}}{2 h}\right) y_{i+1}-\frac{h^{2}}{6} a_{i} y_{i}^{\prime \prime \prime}+ \\
\frac{h^{2}}{12} y_{i}^{(4)}+\tau_{3}=c_{i}
\end{gathered}
$$

where, $\tau_{3}=a_{i} \tau_{1}-\tau_{2}=a_{i} \frac{h^{4} y_{i}^{(5)}}{120}+\frac{h^{4}}{360} y_{i}^{(6)}$

Writing Eq. (3) at discretized mesh, we obtain:

$-y^{\prime \prime}+a_{i} y_{i}^{\prime}+b_{i} y_{i}=c_{i}$

Differentiating Eq. (9) successively, gives

$$
\begin{aligned}
y_{i}^{\prime \prime \prime} & =a_{i} y_{i}^{\prime \prime}+\left(a_{i}^{\prime}+b_{i}\right) y_{i}^{\prime}+b_{i}^{\prime} y_{i}-c_{i}^{\prime} \\
y_{i}^{(4)} & =\left(a_{i}^{2}+2 a_{i}^{\prime}+b_{i}\right) y_{i}^{\prime \prime}+\left(a_{i} a_{i}^{\prime}+a_{i} b_{i}+a_{i}^{\prime \prime}+2 b_{i}^{\prime}\right) y_{i}^{\prime}+\left(b_{i}^{\prime \prime}+a_{i} b_{i}^{\prime}\right) y_{i}-a_{i} c_{i}^{\prime}-c_{i}^{\prime \prime}
\end{aligned}
$$


Substituting Eqs. (10) and (11) into Eq. (8), we obtain:

$$
\begin{aligned}
& \left(-\frac{1}{h^{2}}-\frac{a_{i}}{2 h}\right) y_{i-1}+\left(\frac{2}{h^{2}}+b_{i}+\frac{h^{2}}{12}\left(b_{i}^{\prime \prime}-a_{i} b_{i}^{\prime}\right)\right) y_{i}+\left(-\frac{1}{h^{2}}+\frac{a_{i}}{2 h}\right) y_{i+1}+ \\
& \frac{h^{2}}{12}\left(-a_{i}^{2}+2 a_{i}^{\prime}+b_{i}\right) y_{i}^{\prime \prime}+\frac{h^{2}}{12}\left(-a_{i} a_{i}^{\prime}-a_{i} b_{i}+a_{i}^{\prime \prime}+2 b_{i}^{\prime}\right) y_{i}^{\prime} \\
& =c_{i}-\tau_{3}-\frac{h^{2} a_{i} c_{i}^{\prime}}{12}+\frac{h^{2} c_{i}^{\prime \prime}}{12}
\end{aligned}
$$

Approximating the converted error term, that has the stabilizing effect, in Eq. (12), and by using the central difference formulas given in Eqs. (7) and (8) for $y_{i}^{\prime \prime}$ and $y_{i}^{\prime}$ we obtain:

$$
\begin{aligned}
& \left(-\frac{1}{h^{2}}-\frac{a_{i}}{2 h}+\frac{1}{12}\left(-a_{i}^{2}+2 a_{i}^{\prime}+b_{i}\right)-\frac{h}{24}\left(-a_{i} a_{i}^{\prime}-a_{i} b_{i}+a_{i}^{\prime \prime}+2 b_{i}^{\prime}\right)\right) y_{i-1}+ \\
& \left(\frac{2}{h^{2}}+b_{i}+\frac{h^{2}}{12}\left(b_{i}^{\prime \prime}-a_{i} b_{i}^{\prime}\right)-\frac{1}{6}\left(-a_{i}^{2}+2 a_{i}^{\prime}+b_{i}\right)\right) y_{i}+ \\
& \left(-\frac{1}{h^{2}}+\frac{a_{i}}{2 h}+\frac{1}{12}\left(-a_{i}^{2}+2 a_{i}^{\prime}+b_{i}\right)+\frac{h}{24}\left(-a_{i} a_{i}^{\prime}-a_{i} b_{i}+a_{i}^{\prime \prime}+2 b_{i}^{\prime}\right)\right) y_{i+1} \\
& =c_{i}-\frac{h^{2} a_{i} c_{i}^{\prime}}{12}+\frac{h^{2} c_{i}^{\prime \prime}}{12}+\tau_{4}
\end{aligned}
$$

where, $\tau_{4}$ is local truncation error of fourth order stable central difference method and is given by:

$$
\begin{aligned}
\tau_{4}= & \left(-a_{i}^{\prime} a_{i}+a_{i}^{\prime \prime}-a_{i} b_{i}+2 b_{i}^{\prime}\right) \frac{h^{4} y_{i}^{\prime \prime \prime}}{72}+\left(-a_{i}^{2}+2 a_{i}^{\prime}+b_{i}\right) \frac{h^{4} y_{i}^{(4)}}{144}+ \\
& a_{i} \frac{h^{4} y_{i}^{(5)}}{120}-\frac{h^{4}}{360} y_{i}^{(6)}
\end{aligned}
$$

where, $\tau_{3}=\tau_{2}+a_{i} \tau_{1}=O\left(h^{4}\right)$

From Eq. (13), we get the three-term recurrence relation of the form:

$$
-E_{i} y_{i-1}+F_{i} y_{i}-G_{i} y_{i+1}=H_{i}
$$


where,

$$
\begin{aligned}
E_{i}= & \frac{1}{h}+\frac{1}{2 h}-\frac{1}{12}\left(-a_{i}+a_{i}^{\prime}+b_{i}\right) \\
& +\frac{1}{24}\left(-a_{i} a_{i}^{\prime}-a_{i} b_{i}+a_{i}^{\prime \prime}+b_{i}^{\prime}\right) \\
F_{i}= & b_{i}+\frac{2}{h^{2}}-\frac{1}{6}\left(-a_{i}^{2}+2 a_{i}^{\prime}+b_{i}\right)+\frac{h^{2}}{12}\left(b_{i}^{\prime \prime}-a_{i} b_{i}^{\prime}\right) \\
G_{i}= & \frac{1}{h^{2}}-\frac{a_{i}}{2 h}-\frac{1}{12}\left(-a_{i}^{2}+2 a_{i}^{\prime}+b_{i}\right)-\frac{h}{24}\left(-a_{i} a_{i}^{\prime}-a_{i} b_{i}+a_{i}^{\prime \prime}+2 b_{i}^{\prime}\right) \\
H_{i}= & c_{i}-\frac{h^{2}}{12}\left(a_{i} c_{i}^{\prime}-c_{i}^{\prime \prime}\right) \quad, \quad \text { for } i=1,2,3, \ldots, N-1
\end{aligned}
$$

Eq. (15) can easily be solved by applying Thomas Algorithm.

\section{STABILITY AND CONVERGENCE ANALYSIS}

Remark: Here we shall use the definition of the stability of the difference operator given in (Keller, 1968).

Definition: The linear difference operator $L_{h}($.$) is stable if for sufficiently small h$, there exists a constant $k$, independent of $h$, such that

$$
\left|\omega_{j}\right| \leq k\left\{\max \left(\left|\omega_{o}\right|,\left|\omega_{N}\right|\right)+\max _{1 \leq i \leq N-1}\left|L_{h} \omega_{i}\right|\right\}, j=0,1,2, \ldots . N
$$

For any mesh function, $\left\{\omega_{j}\right\}_{j=0}^{N}$.

Theorem: Under the assumption $b\left(x_{i}\right)=\theta>0$, for $\theta$ is constant $-a_{i}^{2}+2 a_{i}^{\prime}+b_{i} \geq 0$ and

$$
h<\min \frac{2\left(-a_{i}^{2}+2 a_{i}^{\prime}+b_{i}\right)}{\left|-a_{i}\left(a_{i}^{\prime}+b_{i}\right)+a_{i}^{\prime \prime}+2 b_{i}^{\prime}\right|} i=1,2,3 \ldots N-1 .
$$

The difference operator defined on Eq. (15) is stable with $k=\max \left\{1, \frac{1}{\theta}\right\}$

Proof: Let $L_{h}($.$) denote the difference operator on left hand side of Eq. (15) and \omega_{i}$ be any mesh function satisfying:

$$
L_{h}\left(\omega_{i}\right)=H_{i}
$$

If $\max \left|\omega_{i}\right|$ occurs for $\mathrm{i}=0$ or $\mathrm{i}=\mathrm{N}$, then the definition holds, since $k \geq 1$. 
So, assume that $\max \left|\omega_{i}\right|$ occurs for $i=1,2,3, \ldots N-1$

Under the given assumption:

$$
E_{i}>0, G_{i}>0, F_{i} \geq E_{i}+G_{i} \text { and }\left|E_{i}\right| \leq\left|G_{i}\right|
$$

This implies that the tri-diagonal system in Eq. (15) is diagonally dominant and its solution exists, is unique (Greenspan and Casulli, 1988). Then by rearranging the difference Eq. (15) and using the non-negativity of the coefficients, we have:

$$
\begin{aligned}
& F_{i}\left|\omega_{i}\right| \leq E_{i}\left|\omega_{i-1}\right|+G_{i}\left|\omega_{i+1}\right|+\left|H_{i}\right| \\
& \Rightarrow F_{i}\left|\omega_{i}\right| \leq E_{i}\left|\omega_{i-1}\right|+G_{i}\left|\omega_{i+1}\right|+\left|L_{h} \omega_{i}\right|
\end{aligned}
$$

Since $b\left(x_{i}\right)=\theta$ is a constant, by assumption $b^{\prime}\left(x_{i}\right)=0$. Thus, we have:

$$
F_{i}=\frac{2}{h^{2}}-\frac{1}{6}\left(-a_{i}^{2}+2 a_{i}^{\prime}-b_{i}\right)+\theta
$$

From $E_{i}+G_{i}=\frac{2}{h^{2}}-\frac{1}{6}\left(-a_{i}^{2}+2 a_{i}^{\prime}+b_{i}\right)$; and Eqs. (17) and (18), we get:

$$
\begin{aligned}
& \left(\frac{2}{h^{2}}-\frac{1}{6}\left(-a_{i}^{2}+2 a_{i}^{\prime}+b_{i}\right)+\theta\right)\left|\omega_{i}\right| \leq E_{i}\left|\omega_{i-1}\right|+G_{i}\left|\omega_{i+1}\right|+ \\
& \left|L_{h} \omega_{i}\right| \leq\left(E_{i}+G_{i}\right) \max _{1 \leq k \leq N-1}\left|\omega_{k}\right|+\max _{1 \leq k \leq N-1}\left|L_{h} \omega_{k}\right|
\end{aligned}
$$

Since the inequalities in Eq. (19) holds for every $i$, it follows that

$$
\begin{aligned}
& \left(\frac{2}{h^{2}}-\frac{1}{6}\left(-a_{i}^{2}+2 a_{i}^{\prime}+b_{i}\right)+\theta\right) \underset{1 \leq i \leq N-1}{\operatorname{m}} x\left|\omega_{i}\right| \\
& \quad \leq\left(\frac{2}{h^{2}}-\frac{1}{6}\left(-a_{i}^{2}+2 a_{i}^{\prime}+b_{i}\right)\right) \underset{1 \leq k \leq N-1}{\max \left|\omega_{k}\right|+\max _{1 \leq k \leq N-1}\left|L_{h} \omega_{k}\right|}
\end{aligned}
$$

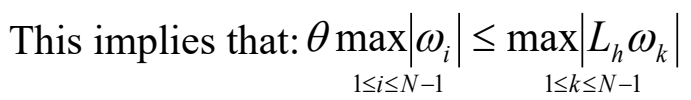

Hence, $\max _{1 \leq i \leq N-1}\left|\omega_{i}\right| \leq \frac{1}{\theta} \max _{1 \leq k \leq N-1}\left|L_{h} \omega_{k}\right| \leq \frac{1}{\theta}\left\{\max \left(\left|\omega_{0}\right|,\left|\omega_{N}\right|\right)+\underset{1 \leq k \leq N-1}{\max }\left|L_{h} \omega_{k}\right|\right\}$

Therefore, $\left|\omega_{i}\right| \leq k\left\{\max \left(\left|\omega_{0}\right|,\left|\omega_{N}\right|\right)+\max _{1 \leq k \leq N-1}\left|L_{k} \omega_{k}\right|\right\}$ 
Hence, $L_{h}($.$) is stable and this implies that the solution to the system of the difference equation Eq. (15) are$ uniformly bounded, independent of mesh size $h$ and the parameter $\varepsilon$.

Hence, the scheme is stable for all step size $h$.

Corollary: Under the conditions for the above theorem, the error $e_{i}=y\left(x_{i}\right)-y_{i}$, between the solution $y(x)$ of the continuous problem and the solution $y_{i}$ of the discrete problem with the boundary condition satisfies the estimate:

$$
\left|e_{i}\right| \leq k \max _{1 \leq i \leq N-1}\left|\tau_{i}\right|
$$

where, $\tau_{4}$ in Eq.(14) denoted by $\tau_{i}$ which is the truncation error given by:

$$
\begin{aligned}
\left|\tau_{i}\right| & \leq \max _{x_{i-1} \leq x \leq x_{i+1}}\left\{\left(-a_{i}^{\prime} a_{i}+a_{i}^{\prime \prime}-a_{i} b_{i}+2 b_{i}^{\prime}\right) \frac{h^{4}\left|y_{i}^{\prime \prime \prime}\right|}{72}\right\} \\
& +\max _{x_{i-1} \leq x \leq x_{i+1}}\left\{\left(-a_{i}^{2}+2 a_{i}^{\prime}+b_{i}\right) \frac{h^{4}\left|y_{i}^{(4)}\right|}{144}\right\} \\
& +\max _{x_{i-1} \leq x \leq x_{i+1}}\left\{a_{i} \frac{h^{4}\left|y_{i}^{(5)}\right|}{120}\right\}+\max _{x_{i-1} \leq x \leq x_{i+1}}\left\{\frac{h^{4}\left|y_{i}^{(6)}\right|}{360}\right\}
\end{aligned}
$$

Proof: Under the given condition error $e_{i}$ satisfies:

$L_{h}\left(e\left(x_{i}\right)\right)=L_{h}\left(y\left(x_{i}\right)-y_{i}\right)=\tau_{i}$ for, $i=1,2,3, \ldots N-1$ and $e_{0}=e_{N}=0$.

Then, from the above theorem stability of $L_{h}($.$) implies that$

$$
\left|y\left(x_{i}\right)-y_{i}\right|=\left|e_{i}\right| \leq k \max _{1 \leq i \leq N-1}\left|\tau_{i}\right|
$$

Hence, the estimate in Eq. (20) establishes the convergence of the scheme for fixed values of the perturbation parameter $\mathcal{\varepsilon}$.

\section{NUMERICAL EXAMPLES}

To demonstrate the applicability of the method, four model singularly perturbed problems have been considered. For each $\varepsilon$ and $\mathrm{N}$, the maximum absolute errors at nodal points are approximated by the formula, $\|E\|=\max \left|y\left(x_{i}\right)-y_{i}\right|$, for $i=0,1,2, \ldots, N$ and where, $y\left(x_{i}\right)$ and $y_{i}$ are the exact and computed solution of the given problem and nodal point $x_{i}$. 
Example 1: Consider the singularly perturbed problem:

$-\varepsilon y^{\prime \prime}+y=f(x), 0 \leq x \leq 1$, with , $y(0)=y(1)=0$

where, $f(x)$ is chosen such that the exact solution is given by:

$y(x)=\exp (x)+e^{\left(\frac{-x}{\sqrt{\varepsilon}}\right)}-x\left(e^{1}+e^{\left(\frac{-1}{\sqrt{\varepsilon}}\right)}\right)-2+2 x$

The numerical solutions in terms of maximum absolute errors are given in

Table 1.

Example 2: Consider the singularly perturbed problem:

$-\varepsilon y^{\prime \prime}+\left(1+x-x^{2}\right) y=f(x), 0 \leq x \leq 1$ with $y(0)=y(1)=0$

where, $f(x)=1+x-x^{2}+\left(2 \sqrt{\varepsilon}-x^{2}+x^{3}\right) e^{\left(-\frac{(1-x)}{\sqrt{\varepsilon}}\right)}+\left(2 \sqrt{\varepsilon}-x(1-x)^{2}\right) e^{\left(\frac{-x}{\sqrt{\varepsilon}}\right)}$

The exact solution is given by:

$y(x)=1+(x-1) e^{\left(\frac{-x}{\sqrt{\varepsilon}}\right)}-x e^{\left(-\frac{(1-x)}{\sqrt{\varepsilon}}\right)}$

The numerical solutions in terms of maximum absolute errors are given in

Table 2.

Example 3: Consider the singularly perturbed problem:

$-\varepsilon\left(\left(1+x^{2}\right) y^{\prime}\right)^{\prime}+\left(1+x-x^{2}\right) y=f(x), 0 \leq x \leq 1$, with $y(0)=y(1)=0 ;$

where, $f(x)$ is chosen such that the exact solution is given by:

$y(x)=1+(x-1) e^{\left(\frac{-x}{\sqrt{\varepsilon}}\right)}-x e^{\left(\frac{x-1}{\sqrt{\varepsilon}}\right)}$

The numerical solutions in terms of maximum absolute errors are given in Table 3 .

Example4: Similarly, consider the singularly perturbed problem:

$-\varepsilon\left(\left(1+x^{2}\right) y^{\prime}\right)^{\prime}+\left(\frac{\cos x}{(3-x)^{3}}\right) y=4\left(3 \mathrm{x}^{2}-3 \mathrm{x}+1\right)\left((\mathrm{x}-1 / 2)^{2}+2\right)$

for $0 \leq x \leq 1$, with $y(0)=-1, \quad y(1)=0$

The exact solution for this problem is not available and its numerical solution is obtained by using the (Doolan et al., 1980). 
The numerical solutions in terms of maximum absolute errors are given in

Table 4.

\section{NUMERICAL RESULTS}

Table 1. Maximum Absolute Errors for Example 1

\begin{tabular}{cccll}
\hline$\varepsilon$ & $N=100$ & $N=500$ & $N=1000$ & $N=1500$ \\
\hline $\begin{array}{c}\text { Our Method } \\
2^{-4}\end{array}$ & $2.0028 \mathrm{E}-09$ & $3.2068 \mathrm{E}-12$ & $2.2249 \mathrm{E}-13$ & $1.3678 \mathrm{E}-13$ \\
$2^{-6}$ & $3.1415 \mathrm{E}-08$ & $5.0189 \mathrm{E}-11$ & $3.1462 \mathrm{E}-12$ & $6.1062 \mathrm{E}-13$ \\
$2^{-8}$ & $5.0141 \mathrm{E}-07$ & $8.0365 \mathrm{E}-10$ & $5.0230 \mathrm{E}-11$ & $9.9172 \mathrm{E}-12$ \\
$2^{-10}$ & $7.9979 \mathrm{E}-06$ & $1.2853 \mathrm{E}-08$ & $8.0358 \mathrm{E}-10$ & $1.5874 \mathrm{E}-10$ \\
$2^{-12}$ & $1.2252 \mathrm{E}-04$ & $2.0554 \mathrm{E}-07$ & $1.2853 \mathrm{E}-08$ & $2.5393 \mathrm{E}-09$ \\
$2^{-14}$ & $1.8953 \mathrm{E}-04$ & $3.2823 \mathrm{E}-06$ & $2.0554 \mathrm{E}-07$ & $4.0615 \mathrm{E}-08$ \\
$2^{-16}$ & $1.6249 \mathrm{E}-03$ & $5.2132 \mathrm{E}-05$ & $3.2823 \mathrm{E}-06$ & $6.4929 \mathrm{E}-07$
\end{tabular}

(Kadalbajoo and Kumar, 2010) using variable mesh

$\begin{array}{lllll}2^{-4} & 2.380 \mathrm{E}-05 & 9.440 \mathrm{E}-07 & 2.360 \mathrm{E}-07 & 1.0500 \mathrm{E}-07 \\ 2^{-6} & 5.310 \mathrm{E}-05 & 2.100 \mathrm{E}-06 & 5.260 \mathrm{E}-07 & 2.3400 \mathrm{E}-07 \\ 2^{-8} & 1.070 \mathrm{E}-04 & 4.260 \mathrm{E}-06 & 1.060 \mathrm{E}-06 & 4.7300 \mathrm{E}-07 \\ 2^{-10} & 2.150 \mathrm{E}-04 & 8.530 \mathrm{E}-06 & 2.130 \mathrm{E}-06 & 9.4800 \mathrm{E}-07 \\ 2^{-12} & 4.310 \mathrm{E}-04 & 1.710 \mathrm{E}-05 & 4.260 \mathrm{E}-06 & 1.9000 \mathrm{E}-06 \\ 2^{-14} & 8.610 \mathrm{E}-04 & 3.410 \mathrm{E}-05 & 8.530 \mathrm{E}-06 & 3.7900 \mathrm{E}-06 \\ 2^{-16} & 1.700 \mathrm{E}-03 & 6.820 \mathrm{E}-05 & 1.710 \mathrm{E}-05 & 7.5800 \mathrm{E}-06\end{array}$

(Kadalbajoo and Kumar, 2010) using Uniform mesh

$\begin{array}{ccccc}2^{-4} & 2.480 \mathrm{E}-05 & 9.900 \mathrm{E}-07 & 2.480 \mathrm{E}-07 & 1.1000 \mathrm{E}-07 \\ 2^{-6} & 9.810 \mathrm{E}-05 & 3.930 \mathrm{E}-06 & 9.820 \mathrm{E}-07 & 4.3600 \mathrm{E}-07 \\ 2^{-8} & 3.910 \mathrm{E}-04 & 1.570 \mathrm{E}-05 & 3.920 \mathrm{E}-06 & 1.7400 \mathrm{E}-06 \\ 2^{-10} & 1.600 \mathrm{E}-03 & 6.270 \mathrm{E}-05 & 1.570 \mathrm{E}-05 & 6.9800 \mathrm{E}-06 \\ 2^{-12} & 5.900 \mathrm{E}-03 & 2.510 \mathrm{E}-04 & 6.270 \mathrm{E}-05 & 2.7900 \mathrm{E}-05 \\ 2^{-14} & 2.150 \mathrm{E}-02 & 9.980 \mathrm{E}-04 & 2.510 \mathrm{E}-04 & 1.1200 \mathrm{E}-04 \\ 2^{-16} & 4.120 \mathrm{E}-02 & 3.900 \mathrm{E}-03 & 9.980 \mathrm{E}-04 & 4.4500 \mathrm{E}-04\end{array}$


Table 2. Maximum Absolute Errors for Example 2

\begin{tabular}{ccccccc}
\hline$\varepsilon$ & $\mathrm{N}=16$ & $\mathrm{~N}=32$ & $\mathrm{~N}=64$ & $N=128$ & $N=256$ & $N=512$ \\
\hline Our Method & & & & & \\
$1 / 8$ & $0.1424 \mathrm{E}-05$ & $0.8806 \mathrm{E}-07$ & $0.5486 \mathrm{E}-08$ & $0.3429 \mathrm{E}-09$ & $0.2141 \mathrm{E}-10$ & $0.1325 \mathrm{E}-11$ \\
$1 / 16$ & $0.4148 \mathrm{E}-05$ & $0.2577 \mathrm{E}-06$ & $0.1617 \mathrm{E}-07$ & $0.1017 \mathrm{E}-08$ & $0.6322 \mathrm{E}-10$ & $0.3933 \mathrm{E}-11$ \\
$1 / 32$ & $0.9622 \mathrm{E}-05$ & $0.5962 \mathrm{E}-06$ & $0.3721 \mathrm{E}-07$ & $0.2324 \mathrm{E}-08$ & $0.10452 \mathrm{E}-09$ & $0.9143 \mathrm{E}-11$ \\
$1 / 64$ & $0.3074 \mathrm{E}-04$ & $0.1927 \mathrm{E}-05$ & $0.1207 \mathrm{E}-06$ & $0.7502 \mathrm{E}-08$ & $0.2443 \mathrm{E}-08$ & $0.2930 \mathrm{E}-10$ \\
$1 / 128$ & $0.1301 \mathrm{E}-03$ & $0.8424 \mathrm{E}-05$ & $0.5255 \mathrm{E}-06$ & $0.3280 \mathrm{E}-07$ & $0.2053 \mathrm{E}-08$ & $0.1283 \mathrm{E}-09$ \\
$1 / 256$ & $0.5910 \mathrm{E}-03$ & $0.3704 \mathrm{E}-04$ & $0.2319 \mathrm{E}-05$ & $0.1450 \mathrm{E}-06$ & $0.9072 \mathrm{E}-08$ & $0.5671 \mathrm{E}-09$ \\
$1 / 512$ & $0.1331 \mathrm{E}-02$ & $0.1444 \mathrm{E}-03$ & $0.9916 \mathrm{E}-05$ & $0.6241 \mathrm{E}-06$ & $0.3905 \mathrm{E}-07$ & $0.2443 \mathrm{E}-08$ \\
$1 / 1024$ & $0.1521 \mathrm{E}-02$ & $0.6190 \mathrm{E}-03$ & $0.4110 \mathrm{E}-04$ & $0.2633 \mathrm{E}-05$ & $0.1640 \mathrm{E}-06$ & $0.1030 \mathrm{E}-07$ \\
$($ Patidar and Kadalbajoo, & $2003) \mathrm{Using}$ & Fitting Factor & & & \\
$1 / 8$ & $0.320 \mathrm{E}-03$ & $0.800 \mathrm{E}-04$ & $0.200 \mathrm{E}-04$ & $0.500 \mathrm{E}-05$ & $0.120 \mathrm{E}-05$ & $0.310 \mathrm{E}-06$ \\
$1 / 16$ & $0.350 \mathrm{E}-03$ & $0.860 \mathrm{E}-04$ & $0.210 \mathrm{E}-04$ & $0.530 \mathrm{E}-05$ & $0.130 \mathrm{E}-05$ & $0.330 \mathrm{E}-06$ \\
$1 / 32$ & $0.400 \mathrm{E}-03$ & $0.990 \mathrm{E}-04$ & $0.250 \mathrm{E}-04$ & $0.620 \mathrm{E}-05$ & $0.150 \mathrm{E}-05$ & $0.390 \mathrm{E}-06$ \\
$1 / 64$ & $0.530 \mathrm{E}-03$ & $0.130 \mathrm{E}-03$ & $0.330 \mathrm{E}-04$ & $0.820 \mathrm{E}-05$ & $0.210 \mathrm{E}-05$ & $0.510 \mathrm{E}-06$ \\
$1 / 128$ & $0.830 \mathrm{E}-03$ & $0.190 \mathrm{E}-03$ & $0.460 \mathrm{E}-04$ & $0.120 \mathrm{E}-04$ & $0.290 \mathrm{E}-05$ & $0.720 \mathrm{E}-06$ \\
$1 / 256$ & $0.130 \mathrm{E}-02$ & $0.260 \mathrm{E}-03$ & $0.660 \mathrm{E}-04$ & $0.160 \mathrm{E}-04$ & $0.410 \mathrm{E}-05$ & $0.100 \mathrm{E}-05$ \\
$1 / 512$ & $0.180 \mathrm{E}-02$ & $0.420 \mathrm{E}-03$ & $0.950 \mathrm{E}-04$ & $0.230 \mathrm{E}-04$ & $0.580 \mathrm{E}-05$ & $0.140 \mathrm{E}-05$ \\
$1 / 1024$ & $0.250 \mathrm{E}-02$ & $0.620 \mathrm{E}-03$ & $0.130 \mathrm{E}-03$ & $0.330 \mathrm{E}-04$ & $0.810 \mathrm{E}-05$ & $0.200 \mathrm{E}-05$ \\
\hline
\end{tabular}

Table 3. Maximum Absolute Errors for Example 3

\begin{tabular}{cccccccc}
\hline$\varepsilon$ & $N=8$ & $\mathrm{~N}=16$ & $\mathrm{~N}=32$ & $\mathrm{~N}=64$ & $N=128$ & $N=256$ & $N=512$ \\
\hline $2^{-3}$ & $1.44 \mathrm{E}-03$ & $3.64 \mathrm{E}-04$ & $9.06 \mathrm{E}-05$ & $2.26 \mathrm{E}-05$ & $5.66 \mathrm{E}-06$ & $1.41 \mathrm{E}-06$ & $3.53 \mathrm{E}-07$ \\
$2^{-4}$ & $2.95 \mathrm{E}-03$ & $5.85 \mathrm{E}-04$ & $1.46 \mathrm{E}-04$ & $3.67 \mathrm{E}-05$ & $9.17 \mathrm{E}-06$ & $2.29 \mathrm{E}-06$ & $5.73 \mathrm{E}-07$ \\
$2^{-5}$ & $3.71 \mathrm{E}-03$ & $8.57 \mathrm{E}-04$ & $2.08 \mathrm{E}-04$ & $5.09 \mathrm{E}-05$ & $1.26 \mathrm{E}-05$ & $3.16 \mathrm{E}-06$ & $7.89 \mathrm{E}-07$ \\
$2^{-6}$ & $3.12 \mathrm{E}-03$ & $9.58 \mathrm{E}-04$ & $2.12 \mathrm{E}-04$ & $6.44 \mathrm{E}-05$ & $1.51 \mathrm{E}-05$ & $3.70 \mathrm{E}-06$ & $9.45 \mathrm{E}-07$ \\
$2^{-7}$ & $2.81 \mathrm{E}-03$ & $1.29 \mathrm{E}-03$ & $2.23 \mathrm{E}-04$ & $6.92 \mathrm{E}-05$ & $1.65 \mathrm{E}-05$ & $4.17 \mathrm{E}-06$ & $1.03 \mathrm{E}-06$ \\
$2^{-8}$ & $4.63 \mathrm{E}-03$ & $1.65 \mathrm{E}-03$ & $2.33 \mathrm{E}-04$ & $6.09 \mathrm{E}-05$ & $1.73 \mathrm{E}-05$ & $4.37 \mathrm{E}-06$ & $1.08 \mathrm{E}-06$ \\
$2^{-12}$ & $6.76 \mathrm{E}-02$ & $3.76 \mathrm{E}-02$ & $7.40 \mathrm{E}-03$ & $6.17 \mathrm{E}-04$ & $3.54 \mathrm{E}-05$ & $4.74 \mathrm{E}-06$ & $1.21 \mathrm{E}-06$ \\
\hline
\end{tabular}


Table 4. Maximum Absolute Errors for Example 4

\begin{tabular}{|c|c|c|c|c|c|c|}
\hline$\varepsilon$ & $\mathrm{N}=32$ & $\mathrm{~N}=64$ & $N=128$ & $N=256$ & $N=512$ & $N=1024$ \\
\hline \multicolumn{7}{|c|}{ Our Method } \\
\hline $2^{-2}$ & 3.7103E-08 & $2.3160 \mathrm{E}-09$ & $1.4471 \mathrm{E}-10$ & $9.0275 \mathrm{E}-12$ & $6.7535 \mathrm{E}-13$ & $8.0491 \mathrm{E}-14$ \\
\hline $2^{-4}$ & $2.7916 \mathrm{E}-07$ & $1.7470 \mathrm{E}-08$ & $1.0916 \mathrm{E}-09$ & $6.8258 \mathrm{E}-11$ & $4.9849 \mathrm{E}-12$ & $4.9531 \mathrm{E}-12$ \\
\hline $2^{-6}$ & $2.8983 \mathrm{E}-06$ & $1.8098 \mathrm{E}-07$ & $1.1309 \mathrm{E}-08$ & $7.0665 \mathrm{E}-10$ & 4.6779E-11 & $7.6206 \mathrm{E}-12$ \\
\hline $2^{-8}$ & $2.8429 \mathrm{E}-05$ & $1.7762 \mathrm{E}-06$ & $1.1100 \mathrm{E}-07$ & $6.9378 \mathrm{E}-09$ & $4.3499 \mathrm{E}-10$ & $2.8159 \mathrm{E}-11$ \\
\hline $2^{-10}$ & $2.2479 \mathrm{E}-04$ & $1.4080 \mathrm{E}-05$ & $8.7980 \mathrm{E}-07$ & $5.4984 \mathrm{E}-08$ & $3.4341 \mathrm{E}-09$ & $2.2168 \mathrm{E}-10$ \\
\hline $2^{-12}$ & $3.1128 \mathrm{E}-03$ & $1.9481 \mathrm{E}-04$ & $1.2190 \mathrm{E}-05$ & 7.6239E-07 & $4.7650 \mathrm{E}-08$ & $2.9734 \mathrm{E}-09$ \\
\hline $2^{-14}$ & $5.0533 \mathrm{E}-02$ & $3.3041 \mathrm{E}-03$ & $2.0803 \mathrm{E}-04$ & $1.3042 \mathrm{E}-05$ & $8.1533 \mathrm{E}-07$ & $5.0976 \mathrm{E}-08$ \\
\hline $2^{-16}$ & $1.7521 \mathrm{E}-01$ & $3.3893 \mathrm{E}-02$ & $3.5055 \mathrm{E}-03$ & $2.2167 \mathrm{E}-04$ & $1.3873 \mathrm{E}-05$ & $8.6815 \mathrm{E}-07$ \\
\hline \multicolumn{7}{|c|}{ (Kadalbajoo and Kumar, 2008) Using Fitting Factor } \\
\hline $2^{-2}$ & $1.310 \mathrm{E}-03$ & $3.280 \mathrm{E}-04$ & $8.210 \mathrm{E}-05$ & $2.050 \mathrm{E}-05$ & $5.130 \mathrm{E}-06$ & $1.280 \mathrm{E}-06$ \\
\hline $2^{-4}$ & $4.930 \mathrm{E}-03$ & $1.230 \mathrm{E}-03$ & $3.080 \mathrm{E}-04$ & $7.710 \mathrm{E}-05$ & $1.930 \mathrm{E}-05$ & $4.820 \mathrm{E}-06$ \\
\hline $2^{-6}$ & $1.600 \mathrm{E}-02$ & $4.000 \mathrm{E}-03$ & $1.000 \mathrm{E}-03$ & $2.500 \mathrm{E}-04$ & $6.260 \mathrm{E}-05$ & $1.560 \mathrm{E}-05$ \\
\hline $2^{-8}$ & $3.710 \mathrm{E}-02$ & $9.270 \mathrm{E}-03$ & $2.320 \mathrm{E}-03$ & $5.790 \mathrm{E}-04$ & $1.450 \mathrm{E}-04$ & $3.620 \mathrm{E}-05$ \\
\hline $2^{-10}$ & $6.190 \mathrm{E}-02$ & $1.540 \mathrm{E}-02$ & $3.860 \mathrm{E}-03$ & $9.650 \mathrm{E}-04$ & $2.410 \mathrm{E}-04$ & $6.030 \mathrm{E}-05$ \\
\hline $2^{-12}$ & $9.390 \mathrm{E}-02$ & $2.340 \mathrm{E}-02$ & $5.830 \mathrm{E}-03$ & $1.460 \mathrm{E}-03$ & $3.640 \mathrm{E}-04$ & $9.100 \mathrm{E}-05$ \\
\hline $2^{-14}$ & $1.340 \mathrm{E}-01$ & $3.290 \mathrm{E}-02$ & $8.150 \mathrm{E}-03$ & $2.030 \mathrm{E}-03$ & $5.080 \mathrm{E}-04$ & $1.270 \mathrm{E}-04$ \\
\hline $2^{-16}$ & $1.900 \mathrm{E}-01$ & $4.310 \mathrm{E}-02$ & $1.050 \mathrm{E}-02$ & $2.600 \mathrm{E}-03$ & $6.500 \mathrm{E}-04$ & $1.620 \mathrm{E}-04$ \\
\hline
\end{tabular}

The computational rate of convergence can also be obtained by using the double mesh principle, defined as follow:

Let $Z_{h}=\max _{i}\left|y_{i}^{h}-y_{i}^{h / 2}\right|, \quad i=1,2, \ldots, N-1$

Where, $y_{i}^{h}$ is the numerical solution on the mesh $\left\{x_{i}\right\}_{1}^{N-1}$ at the nodal point $x_{i}$ and $x_{i}=x_{0}+h$, $i=1,2, \ldots, N-1$,

$y_{i}^{h / 2}$ is the numerical solution at the nodal point $x_{i}$ on the mesh $\left\{x_{i}\right\}_{1}^{2 N-1}$ where, $x_{i}=x_{0}+h / 2, \quad i=1,2, \ldots, 2 N-1$.

In the same way one can define $Z_{h / 2}$ by replacing $h$ by $h / 2$ and $N-1$ by $2 N-1$. 
That is, $Z_{h / 2}=\max _{i}\left|y_{i}^{h / 2}-y_{i}^{h / 4}\right|$, for $i=1,2, \ldots, 2 N-1$.

The computed rate of convergence is defined as:

Rate $=\log \left(\frac{Z_{h}-Z_{h / 2}}{2}\right)$

Also maximum absolute error based on the double-mesh principle

(Doolan et.al., 1980), is given by:

$\|E\|=\max _{i}\left|y_{i}{ }^{N}-y_{2 i}{ }^{2 N}\right|$, for $i=0,1,2, \ldots N$. and $y_{i}^{h / 2}$ denotes the values of $y_{i}$ for mesh length $\frac{h}{2}$.

The rate of convergence for Examples 1 and 4, which have been considered as sample, is presented in Table 5 .

Table 5. Rate of convergence of Example 1 and 4

\begin{tabular}{|c|c|c|c|c|c|c|c|}
\hline & $\varepsilon$ & $h$ & $h / 2$ & $Z_{h}$ & $h / 4$ & $Z_{h / 2}$ & Rate \\
\hline \multirow{9}{*}{ 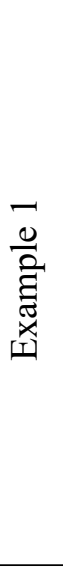 } & \multirow[t]{3}{*}{$2^{-4}$} & $1 / 100$ & $1 / 200$ & $1.877660 \mathrm{E}-09$ & $1 / 400$ & $1.173327 \mathrm{E}-10$ & 4.00006 \\
\hline & & $1 / 200$ & $1 / 400$ & $1.173327 \mathrm{E}-10$ & $1 / 800$ & $7.318690 \mathrm{E}-12$ & 4.00008 \\
\hline & & $1 / 400$ & $1 / 800$ & $7.318690 \mathrm{E}-12$ & $1 / 1600$ & $4.35097 \mathrm{E}-13$ & 4.00010 \\
\hline & \multirow[t]{3}{*}{$2^{-8}$} & $1 / 100$ & $1 / 200$ & 4.700047E-07 & $1 / 400$ & 1.83953E-09 & 3.999227 \\
\hline & & $1 / 200$ & $1 / 400$ & $2.940009 \mathrm{E}-08$ & $1 / 800$ & $1.14926 \mathrm{E}-10$ & 3.998824 \\
\hline & & $1 / 400$ & $1 / 800$ & $1.839530 \mathrm{E}-09$ & $1 / 1600$ & $1.14926 \mathrm{E}-10$ & 4.000056 \\
\hline & \multirow[t]{3}{*}{$2^{-12}$} & $1 / 100$ & $1 / 200$ & $1.145221 \mathrm{E}-04$ & $1 / 400$ & 7.49654E-06 & 3.933614 \\
\hline & & $1 / 200$ & $1 / 400$ & 7.496540E-06 & $1 / 800$ & $4.70000 \mathrm{E}-07$ & 3.995819 \\
\hline & & $1 / 400$ & $1 / 800$ & $4.700000 \mathrm{E}-07$ & $1 / 1600$ & 2.93981E-08 & 3.999216 \\
\hline \multirow{11}{*}{ 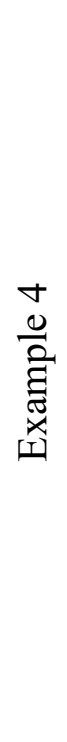 } & \multirow[t]{3}{*}{$2^{-4}$} & $1 / 32$ & $1 / 64$ & $2.6169 \mathrm{E}-07$ & $1 / 128$ & $1.6378 \mathrm{E}-08$ & 3.99870 \\
\hline & & $1 / 64$ & $1 / 128$ & $1.6378 \mathrm{E}-08$ & $1 / 256$ & $1.0233 \mathrm{E}-09$ & 4.00041 \\
\hline & & $1 / 128$ & $1 / 256$ & 1.0233E-09 & $1 / 512$ & $6.3273 \mathrm{E}-11$ & 4.00016 \\
\hline & \multirow[t]{4}{*}{$2^{-8}$} & $1 / 32$ & $1 / 64$ & $2.6653 \mathrm{E}-05$ & $1 / 128$ & $1.6652 \mathrm{E}-06$ & 4.00054 \\
\hline & & $1 / 64$ & $1 / 128$ & $1.6652 \mathrm{E}-06$ & $1 / 256$ & $1.0406 \mathrm{E}-07$ & 4.00023 \\
\hline & & $1 / 128$ & $1 / 256$ & $1.0406 \mathrm{E}-07$ & $1 / 512$ & $6.5028 \mathrm{E}-09$ & 4.00021 \\
\hline & & $1 / 256$ & $1 / 512$ & $6.5028 \mathrm{E}-09$ & $1 / 1024$ & $4.0683 \mathrm{E}-10$ & 3.99862 \\
\hline & \multirow[t]{4}{*}{$2^{-12}$} & $1 / 32$ & $1 / 64$ & 0.0029000 & $1 / 128$ & $1.8262 \mathrm{E}-04$ & 3.99812 \\
\hline & & $1 / 64$ & $1 / 128$ & $1.8262 \mathrm{E}-04$ & $1 / 256$ & $1.1428 \mathrm{E}-05$ & 3.99812 \\
\hline & & $1 / 128$ & $1 / 256$ & $1.1428 \mathrm{E}-05$ & $1 / 512$ & $7.1474 \mathrm{E}-07$ & 3.99901 \\
\hline & & $1 / 256$ & $1 / 512$ & 7.1474E-07 & $1 / 1024$ & 4.4677E-08 & 3.99984 \\
\hline
\end{tabular}


The following graphs (Figures 1-4), show the numerical solutions obtained by the present method for $h>\varepsilon$.

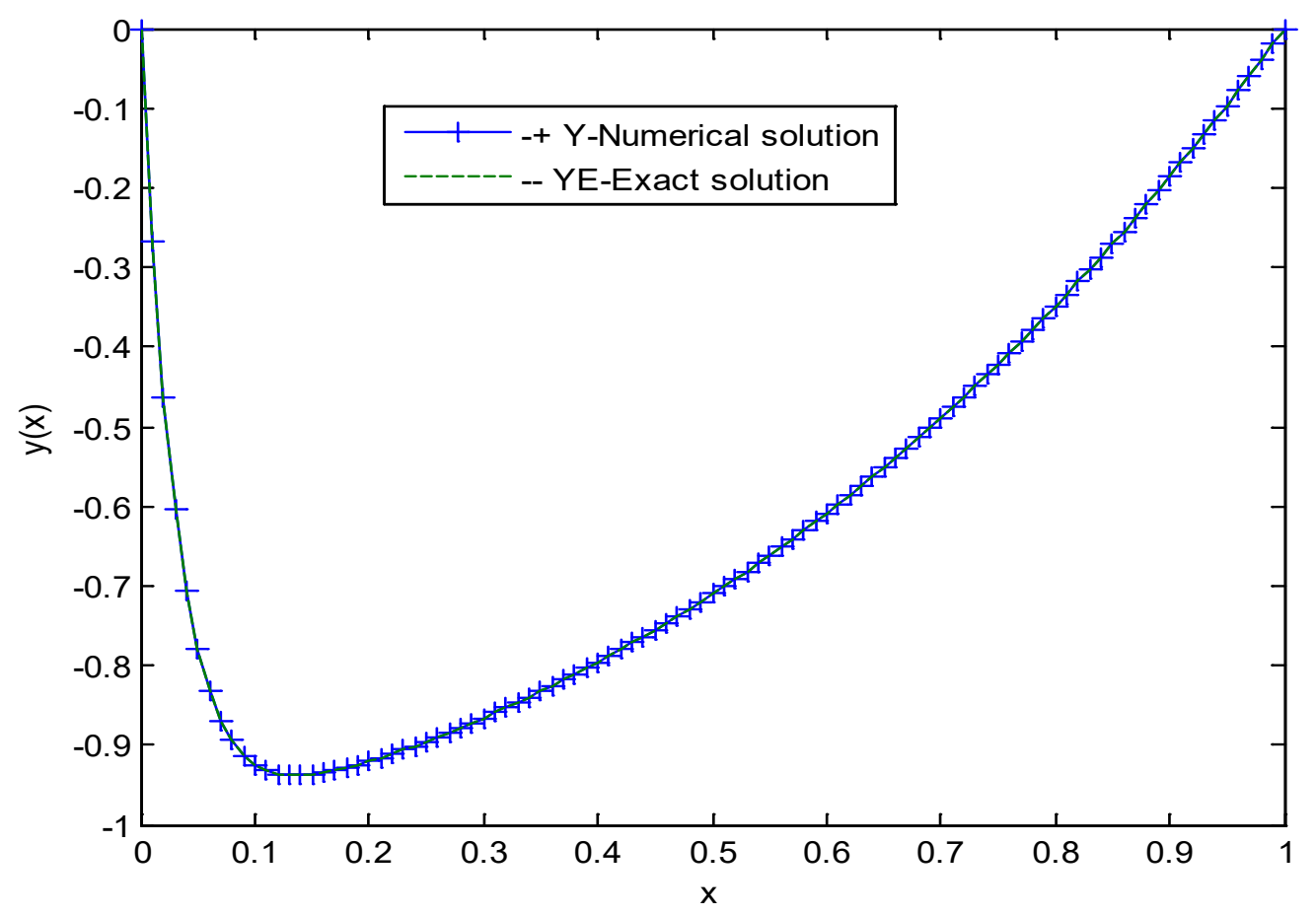

Figure 1. Numerical solution of Example 1 with $\varepsilon=0.001$ and $\mathrm{h}=0.01$

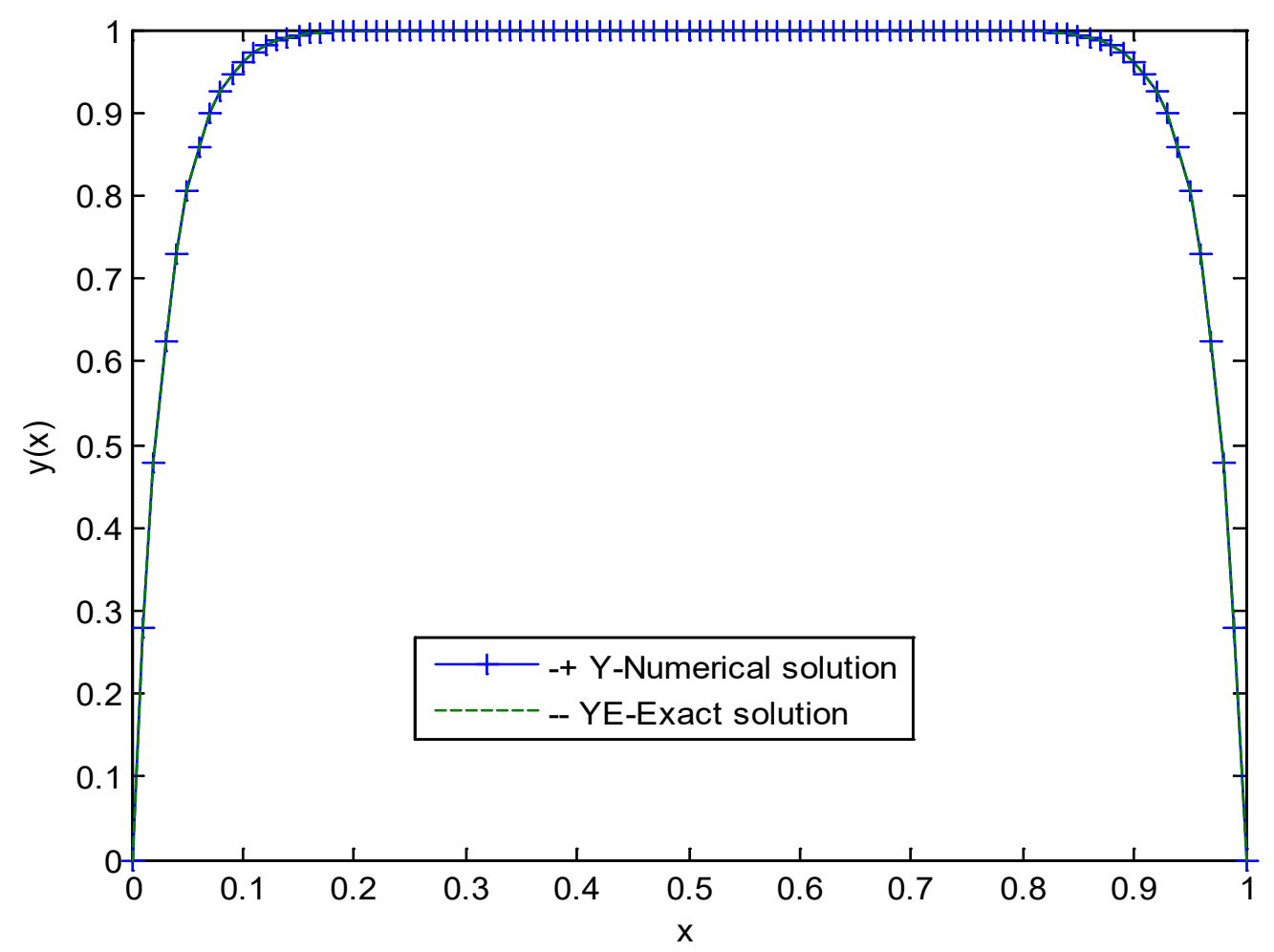

Figure 2. Numerical solution of Example 2 with $\varepsilon=0.001$ and $\mathrm{h}=0.01$ 


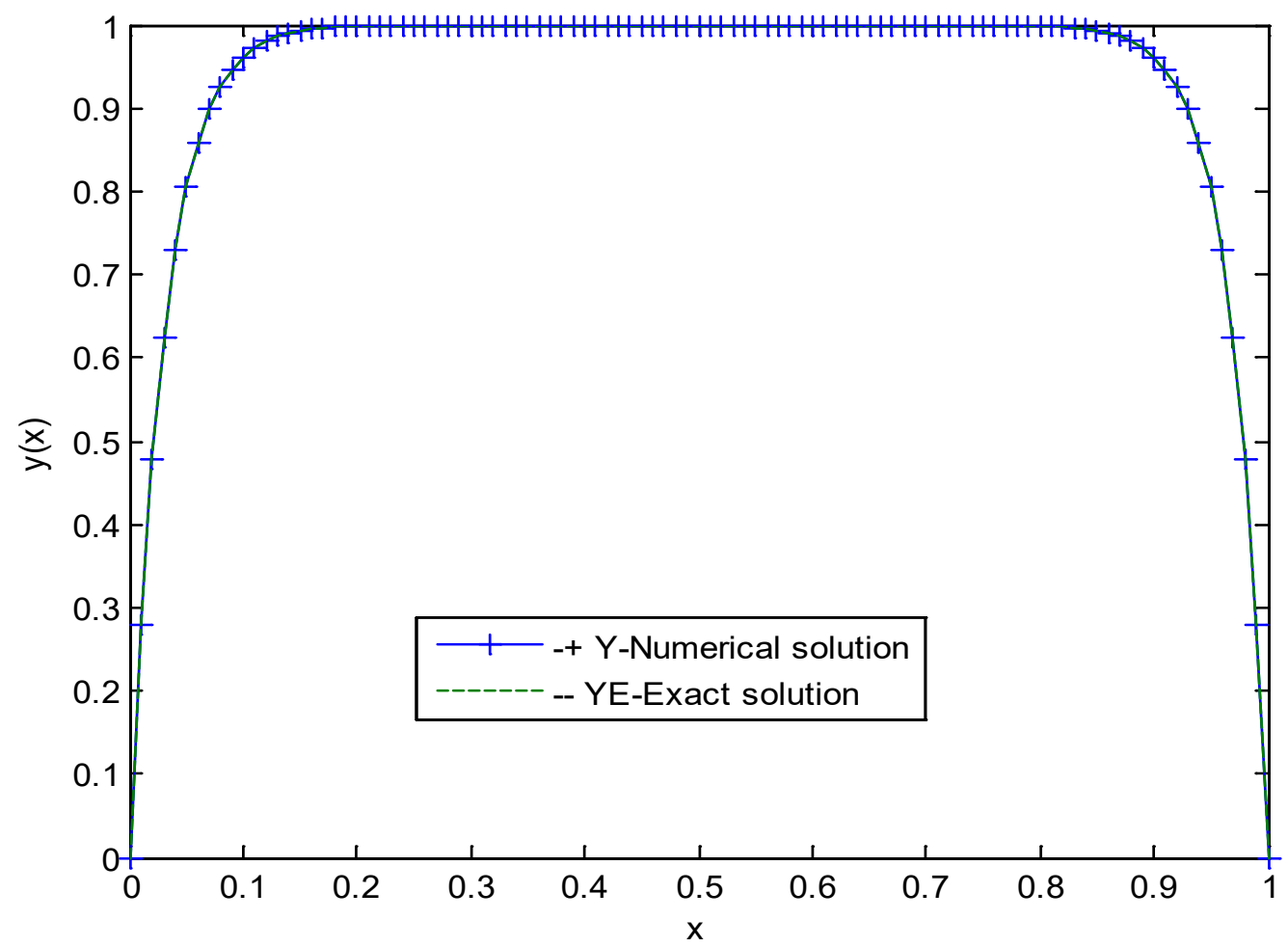

Figure 3. Numerical solution of Example 3 with $\varepsilon=0.001$ and $\mathrm{h}=0.01$

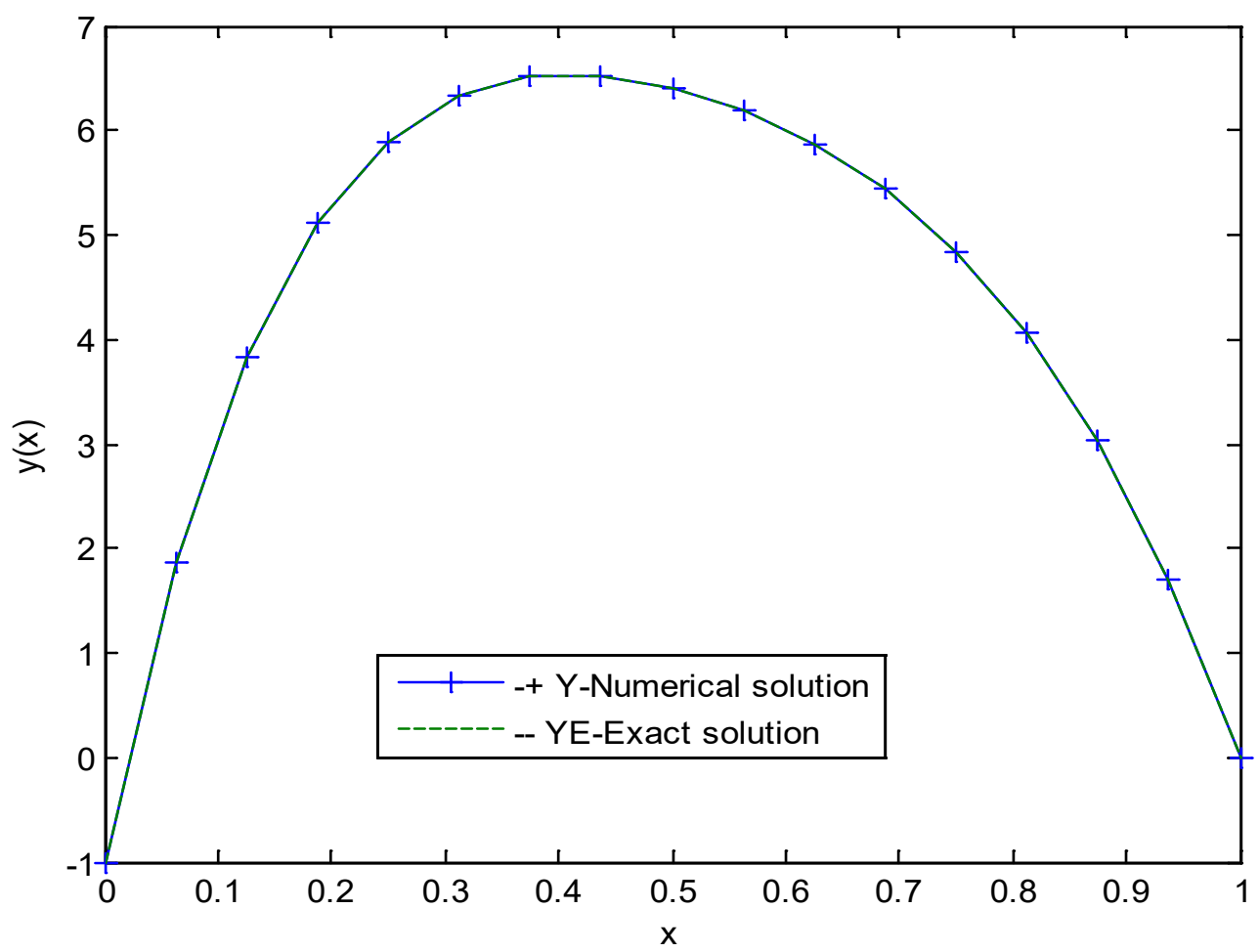

Figure 4. Numerical solution of Example 4 with $\varepsilon=1 / 32$ and $\mathrm{h}=1 / 16$ 


\section{DISCUSSION AND CONCLUSION}

Fourth order stable central difference method has been presented for solving self-adjoint singularly perturbed two point boundary value problems. Four examples are given to demonstrate the efficiency of the proposed method. The maximum absolute errors at different nodal points are tabulated in the tables (Tables 1-4) for different values of the perturbation parameter $\varepsilon$ and compared with some previous findings of other methods reported in the literature. The numerical results presented in Tables 1, 2 and 4 clearly indicate that the proposed scheme is more efficient than the methods given in (Patidar and Kadalbajoo, 2003; Kumar and Kadalbajoo, 2008; Kadalbajoo and Kumar, 2010). Table 3 also shows that the maximum absolute error decreases as the mesh size $h$ decreases, which in turn shows the convergence of the computed solution and it also substantiates the theoretical convergence analysis given. To further corroborate the applicability of the proposed method, graphs between exact solution and approximate solutions have been plotted (Figures 1-4) for $\varepsilon=10^{-3}, \varepsilon=1 / 32, h=10^{-2}$ and $h=1 / 16$. It is observed that the numerical solutions are in a very good agreement with the exact solution for small value of $\varepsilon$ (i.e. $h \geq \varepsilon$ ) for which most of classical numerical methods fail to give good result. The stability estimates are discussed and the computational order of convergence is also calculated (Table 5). In a concise manner, the present method approximates the exact solution very well and gives better results than some existing methods reported in the literature.

\section{ACKNOWLEDGMENTS}

The authors would like to thank Jimma University for the financial and materials support.

\section{REFERENCES}

Boglaev, I. P. (1981). A variation difference scheme for boundary value problems with a small parameter in the highest derivative. Journal of Computational Mathematics and Mathematical Physics 21 (4): $71-81$.

Byme, C and Mishra, H. K. (2009). Notes on self-adjoint differential equations. Computer Technology and Applications 2 (5): 1426-1431.

Doolan, E. P., Miller J. J. H and Schilders W. H. A. (1980). Uniform Numerical Methods for Problems with Initial and Boundary Layers. Dublin, Boole Press.

Farrell, A. F., Miller, J. J. H., O’Riordan, E and Shishkin, G. I. (2000). Robust Computational Techniques for Boundary Layers. Chapman-Hall/CRC, New York.

Greenspan, D and Casulli, V. (1988). Numerical Analysis for Applied Mathematics, Science and Engineering. Addison-Wesley Publishing Co., Inc.

Gupta,Y and Pankaj, S. A. (2011). Computational method for solving two point boundary problems of order four. International Journal of Computer Technology and Applications 2 (5): 1426-1431.

Kadalbajoo, M. K and Kumar. D. (2010).Variable mesh finite difference method for self-adjoint singularly perturbed two-point boundary value problems. Journal of Computational Mathematics 28 (5): 711 724.

Kadalbajoo, M. K and Kumar, D. (2008). Parameter-uniform fitted operator B-spline collocation method for self-adjoint singularly perturbed two-point boundary value problems. Electronic Transactions on 
Numerical Analysis 30: 346-358.

Keller, H. B. (1968). Numerical Methods for Two Point Boundary Value Problems. CBMS-NSF Regional Conference Series in Applied Mathematics. Blaisdel Publishing Company, Waltham.

Mishra, H. K., Kumar, M and Singh, P. (2009). Initial value technique for self-adjoint perturbation boundary value problems. Computational Mathematics and Modeling 20 (2): 207-217.

Patidar, C and Kadalbajoo, M. K. (2003). Exponentially fitted spline approximation method for solving self-adjoint singularly perturbed problem. International Journal of Mathematics and Mathematical Science 61: 3873-3891.

Roos, H. G., Stynes, M and Tobiska, L. (1996). Numerical Methods for Singularly Perturbed Differential Equations: Convection-Diffusion and Flow Problems. Springer Verlag.

Saini, S and Mishra, H. K. (2015). A new quartic B-spline method for third order self-adjoint singularly perturbed boundary value problems. Applied Mathematical Sciences 9 (8): 399- 408. 